\title{
Clinical presentation of a myocardial rhabdomyosarcoma
}

\author{
Michael V. O'Reilly, ${ }^{1}$ Roderick T. McDonald, and V. L. Fornasier \\ From the Division of Cardiology, and the Department of Medicine, Wellesley Hospital, Toronto
}

A case initially diagnosed as tricuspid insufficiency in which critical review of the clinical findings pointed to a diagnosis of pulmonary outflow obstruction is described. At necropsy a myocardial rhabdomyosarcoma was found with associated thromboembolism. The cardinal features were the rapid development of a loud systolic murmur, clinical evidence of right ventricular enlargement and failure with large jugular presystolic ' $a$ ' waves, and a reduction in the intensity of the pulmonary component of the second sound, a shift in the QRS axis to the right, and an increase in the cardiothoracic ratio.

There have been 25 previously reported cases of rhabdomyosarcoma of the heart and though there are no pathognomonic clinical features (Sochocky, I97I), there are a number of manifestations that occur relatively frequently (Hallermann et al., 1963), especially when there is associated pulmonary outflow obstruction, that are suggestive of the diagnosis. In this report we wish to emphasize the importance of these clinical manifestations pointing towards the diagnosis of pulmonary outflow obstruction, which in our case was confirmed (at necropsy) to be secondary to a myocardial rhabdomyosarcoma and thromboembolism.

\section{Case report}

A 52-year-old white man with maturity onset diabetes and chronic lymphocytic leukaemia was admitted to the hospital because of recent development of right heart failure, without haemoptysis, orthopnoea pulmonary congestion, or evidence of mitral valve disease.

Examination revealed a cachetic, dyspnoeic white man with prominent bilateral neck pulsation, blood pressure ro5/75 (13.9/9.9); pulse 88/min, regular, of good volume. More detailed examination of the neck revealed that the pulsation was venous (later determined to be caused by prominent presystolic ' $a$ ' waves, with only small ' $v$ ' waves). The apical impulse was diffuse and displaced almost to the left anterior axillary line, and a left parasternal impulse was palpable. On initial examination, the heart sounds were unremarkable, but there was a grade $3 / 6$ blowing systolic murmur audible over most of the praecordium, maximal over the left sternal edge, and accentuated with inspiration. These findings had

${ }^{1}$ Present address: Cardiac Catheterization Laboratory, Beth Israel'Medical Center, New York, New York ro003, U.S.A. not been present three months previously. The lungs were clear, the liver edge was just palpable, non-tender, and possibly pulsatile. There was moderate bilateral ankle oedema. Electrocardiogram showed sinus rhythm with right axis deviation, not present previously (Fig. I). A chest $x$-ray showed a recent increase in cardiothoracic ratio.

The initial diagnosis was tricuspid insufficiency, cause unknown. Subsequently it was noted that the first heart sound was diminished, especially at the lower sternum, but that the pulmonary component of the second sound was not increased, and that there was a prominent rightsided fourth heart sound. It was also demonstrated that the venous impulse was an ' $a$ ' wave and not $a$ ' $v$ ' wave as previously suspected. There was no ejection sound, opening snap, or diastolic murmur. In view of these observations the diagnosis of tricuspid insufficiency was not substantiated, but no explanation was apparent for the features of right ventricular hypertrophy, in the absence of the usual signs of pulmonary hypertension, left-to-right shunt, or mitral valve disease. Blood cultures were negative.

The patient continued to deteriorate slowly and arrested, sixteen days later, before cardiac catheterization could be arranged. Resuscitation was unsuccessful. Previous treatment had consisted of digoxin $0.25 \mathrm{mg}$ daily, frusemide $40 \mathrm{mg}$ daily, two 'Slow $\mathrm{K}$ ' tablets three times a day, and sulphinpyrazone $200 \mathrm{mg}$ three times a day.

At necropsy the heart weighed $500 \mathrm{~g}$ and the external appearance of the heart was normal. The right atrium was moderately distended but only slight hypertrophy of the right atrial or ventricular wall was present. Arising on the lateral aspect of the infundibular part of the right ventricle there was a smooth surfaced mass with a base covering an area $2 \times 3 \mathrm{~cm}$ and with a tongue extending across the pulmonary valve into the main pulmonary artery. The lumen of pulmonary valve 

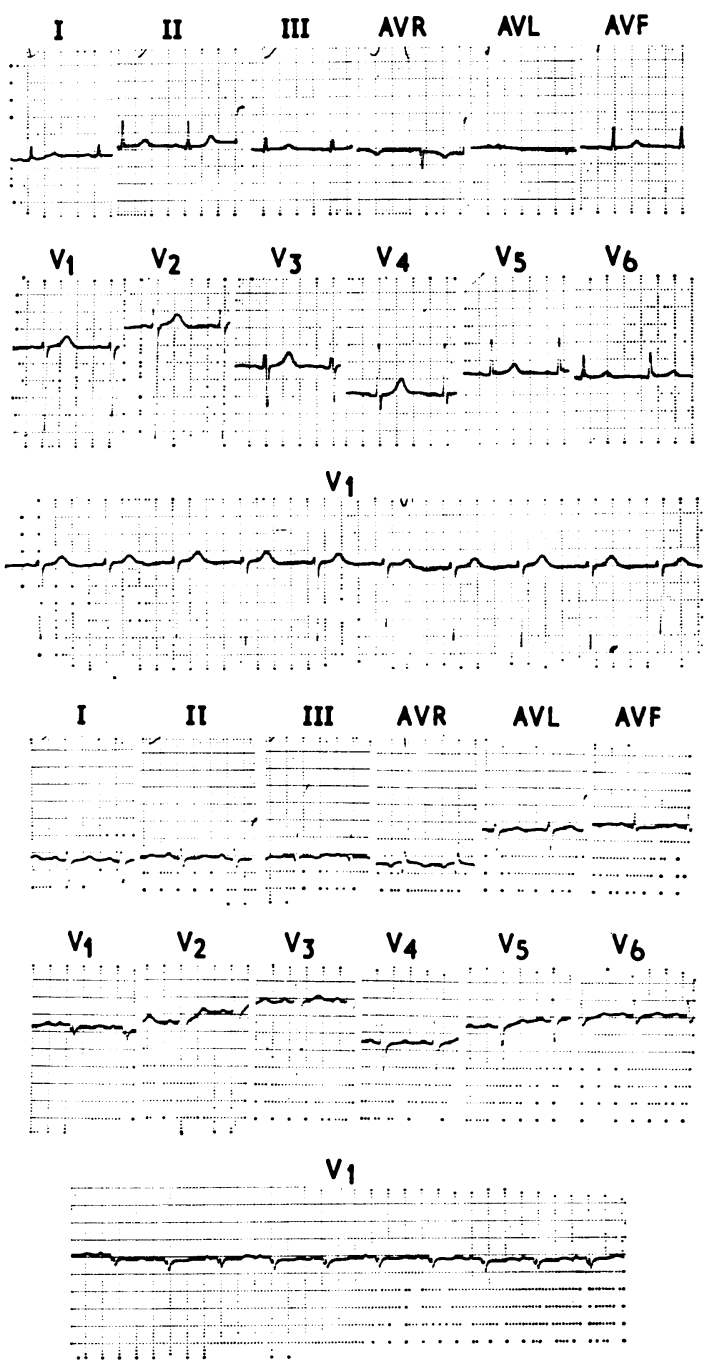

FIG. I Representative electrocardiograms (note axis shift from $+70^{\circ}$ to $\left.+120^{\circ}\right)$.

and artery consisted of a slit-like crevice between the tumour mass and the opposing endothelial surface. Superficial erosion and thrombus at varying stages of organization extended across the open surface of the tumour. Tumour extended almost through the entire thickness of the myocardium of the right ventricle. Masses of tumour presumed to be embolic in nature occluded the main left pulmonary artery and its primary branches. This was associated with further thrombosis within these vessels and beyond. A moderate degree of congestion and vascular engorgement was present throughout the left lung but no frank infarction was present. A serous effusion of $200 \mathrm{ml}$ was present in the left pleural cavity. Microscopical examination of the tumour mass (Fig. 2) showed a spindle cell sarcoma with a moderate degree of cellular pleomorphism and a distinct increase in mitotic activity. There were frequent large strap-like cells with abundant eosinophilic cytoplasm and open-faced irregular nuclei. Occasional cells with distinct cross-striations and the presence of demonstrable myofibrillar material within the large cells with abundant cytoplasm confirmed the diagnosis of rhabdomyosarcoma. The pattern of growth of this tumour within the myocardium and its extension into the pulmonary artery were felt to constitute satisfactory evidence for a primary lesion of the myocardium. A thorough examination revealed no evidence of an additional primary tumour.

Also observed at necropsy was the presence of severe generalized atherosclerosis. There were severe combined ulcero-fibrotic lesions of the aorta and plaques which almost completely occluded the main trunks of the coronary arteries. Of further note was the presence of granular cell tumours (myoblastomata) of the oesophagus and colon. Though in the past these lesions were regarded as originating in muscular tissue, current opinion is in favour of a nerve tissue origin.

From the anatomical findings it was concluded that death resulted from right ventricular failure consequent on the obstruction of the pulmonary valve and artery by the rhabdomyosarcoma of the right ventricle.

\section{Discussion}

This patient with stable, chronic lymphocyctic leukaemia until one month before admission had been responding well to treatment. In the absence of clinical evidence of pulmonary hypertension, mitral valve disease, pulmonary emboli, or tricuspid regurgitation, it was difficult to explain the rapid onset of right-sided heart failure with a progressively downhill course in spite of adequate therapy. This picture of rapid progression in cases of rhabdomyosarcoma was also noted by Hallermann et al. (1963), in 12 patients who had sarcomas involving the pulmonary outflow tract. Pleural and pericardial effusions and a systolic murmur over the sternum or praecordium were also present in many instances. In reporting his case, Hallermann remarked on the rapid progression of the illness, and the poor response to digitalis and diuretics. The signs in our patient were initially thought to be due to tricuspid regurgitation, but this interpretation could not be reconciled with the venous presystolic waves, only small brief ' $v$ ' waves, and the absence of a pulsatile liver; the absence of a loud pulmonary second sound or ejection click (despite the presence of ' $a$ ' waves and a definite fourth heart sound) tended to rule out pulmonary hypertension. The importance of a diminished pulmonary second sound, with evidence of right ventricular hypertrophy as suggestive of pulmonary outflow obstruction, was noted by Green, Crevasse, and Shanklin 

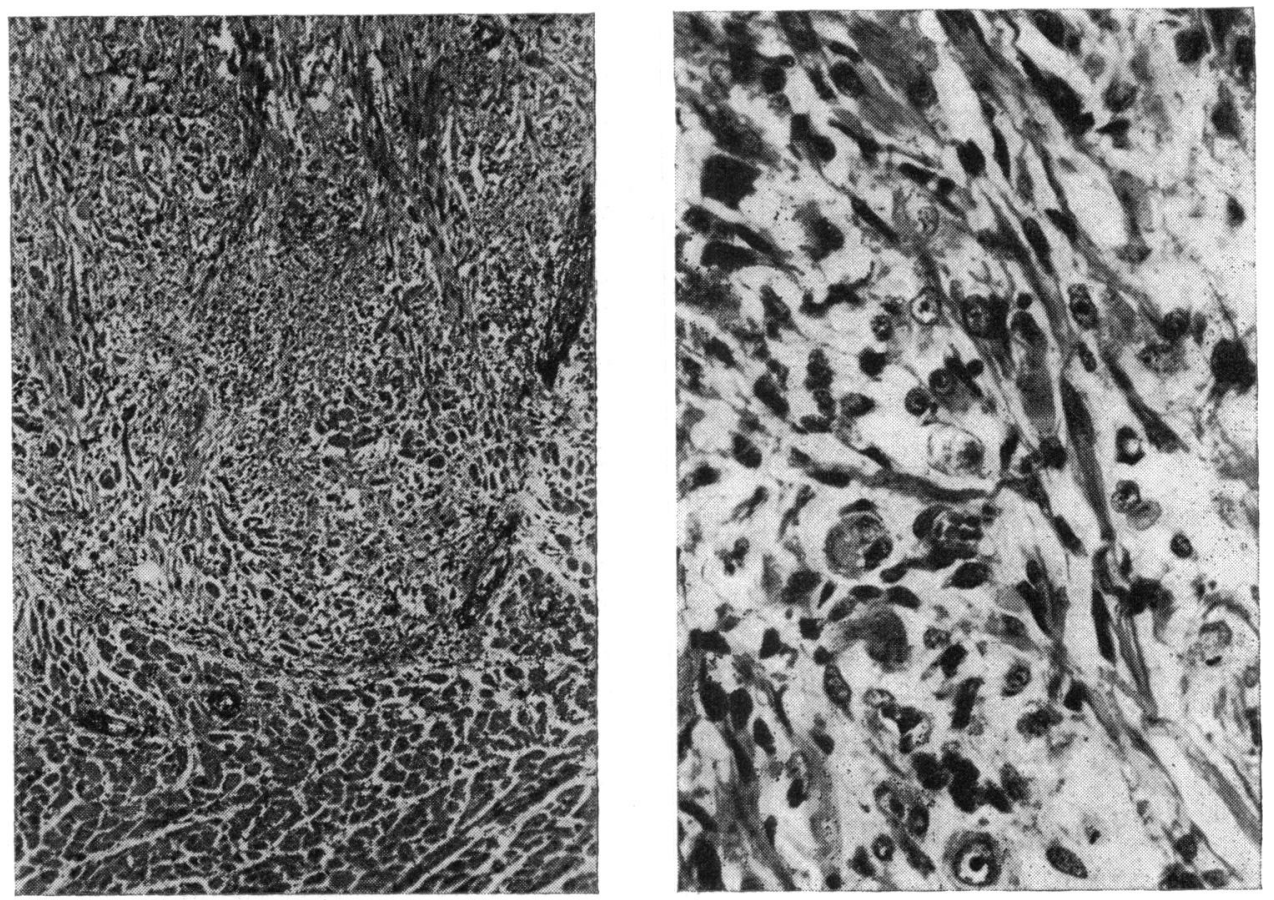

FIG. 2 Left: tumour infiltrating myocardium. ( $\times$ I 56.) Right: histology of rhabdomyosarcoma showing large strap cells with fibrillar material in the abundant cytoplasm and one cell near the centre with characteristic cross-striations. ( $\times$ 250.)

(1964), as well as by Hallermann et al. It was difficult to make a firm diagnosis before death, and though a cardiac tumour was mentioned as part of the differential diagnosis, it was never seriously considered because of its rarity.

Thromboembolism of the right ventricle was not considered clinically, as the first such report (Spencer, King, and Grossmann, 197I) did not appear until three months after our patient died. This patient with a combination of a rhabdomyosarcoma with thromboembolism of the right ventricle and outflow tract, we believe is the first such recorded case. In retrospect, the clinical signs were sufficient to lead to suspicion, as they could not be explained adequately by either tricuspid regurgitation, pulmonary hypertension, or mitral disease.

The incidence of primary, malignant cardiac tumours at routine necropsy is only 0.0017 to 0.05 per cent (Lymburner, 1934; Straus and Merliss, 1945), rhabdomyosarcoma being rare within this group, and the most consistent findings are a rapid onset of right ventricular failure (often unresponsive to medical treatment), a systolic murmur, and the presence of pericardial and pleural effusions, all without obvious cause.

Sudden death is common with these tumours, and may follow intermittent or persistent circulatory failure, or occur without preliminary circulatory symptoms (Friedberg, 1966). It is frequently caused by complete heart block, ventricular fibrillation, or outflow occlusion. Spencer et al. (I97I) noted similar findings in their case of thromboembolism of the right ventricle.

An understandable error is to diagnose tricuspid incompetence or pulmonary hypertension, but this trap can usually be avoided by noting that the venous pulsation in the neck is caused by the presence of presystolic (' $a$ ' waves) rather than sustained ' $c v$ ' waves (which are systolic), that the pulmonary second sound is reduced or absent, and that there is no ejection sound, despite signs of right ventricular enlargement. In this clinical setting the patient should be vigorously investigated to rule out a myocardial tumour or right ventricular thromboembolism, as these conditions may potentially be alleviated by early surgical intervention. 


\section{References}

Friedberg, C. K. (1966). Cardiac tumors. In Diseases of the Heart, 3rd ed., p. 1713. Saunders, Philadelphia and London.

Green, J. R., Crevasse, L. E., and Shanklin, D. R. (1964). Fibromyxosarcoma of the pulmonary artery. Associated with syncope, intractable heart failure, polycythemia and thrombocytopenia. American fournal of Cardiology, I3, 547.

Hallermann, F. J., Kincaid, O. W., Brown, A. L., and Daugherty, G. W. (1963). Rhabdomyosarcoma of the heart producing right ventricular outflow obstruction. An unusual case diagnosed by selective angiocardiography. fournal of the American Medical Association, 184, 939.
Lymburner, R. M. (1934). Tumours of the heart, histopathological and clinical study. Canadian Medical Association fournal, 30, 368.

Sochocky, S. (I97I). Rhabdomyosarcoma of the heart. Minnesota Medicine, 54, 747.

Spencer, J. D., King, J. F., and Grossmann, A. E. (I97I). Cardiac embolus. American Heart fournal, 82, 802.

Straus, R., and Merliss, R. (1945). Primary tumor of the heart. Archives of Pathology, 39, 74.

Requests for reprints to Dr. Michael V. O'Reilly, Cardiac Catheterization Laboratory, Beth Israel Medical Center, ro Nathan D. Perlman Place, New York, N.Y. 10003, U.S.A. 